\title{
Erratum to: Chapter 1 in Pre- and Periprocedural Planning and Patient Management for Spine or Rib Biopsies
}

\author{
Richard Silbergleit and A. Orlando Ortiz
}

\section{Erratum to:}

Chapter 1 in A. Orlando Ortiz, Image-Guided Percutaneous Spine Biopsy.

DOI: 10.1007/978-3-319-43326-4_1.

The first author information on the chapter opening page of chapter 1 was incorrect.

First author for this chapter is:

Richard Silbergleit

The updated version of the original chapter can be found under DOI 10.1007/978-3-319-43326-4_1 